\title{
The Effect of Plate Discretization on Accuracy of the Sound Radiation Efficiency Measurements
}

\author{
Karolina KOLBER $^{(1)}$, Anna SNAKOWSKA ${ }^{(1)}$, Michał KOZUPA ${ }^{(2)}$ \\ (1) AGH University of Science and Technology, Faculty of Mechanical Engineering and Robotics, \\ Department of Mechanics and Vibroacoustics
}

Al. A. Mickiewicza 30, 30-059 Kraków, Poland; e-mail: \{karolina.kolber, anna.snakowska\}@agh.edu.pl

(2) ABB Sp. z o.o. Corporate Research

Starowislna 13A, 31-038 Kraków, Poland; e-mail: Michal.Kozupa@pl.abb.com

(received March 3, 2014; accepted August 24, 2014)

\begin{abstract}
This paper deals with the problem of the effect of discretization level and certain other parameters characterizing the measurement setup on accuracy of the process of determination of the sound radiation efficiency by means of the Discrete Calculation Method (DCM) described by Hashimoto (2001).

The idea behind DCM consists in virtual division of an examined sound radiating structure into rectangular elements each of which is further assumed to contribute to the total radiation effect in the same way as a rigid circular piston having the surface area equal to this of the corresponding virtual element and vibrating in an infinite rigid baffle. The advantage of the method over conventional sound radiation efficiency measurement techniques consists in the fact that instead of acoustic pressure values, source (plate) vibration velocity amplitude values are measured in a selected number of regularly distributed points. In many cases, this allows to determine the sound radiation efficiency with sufficient accuracy, especially for the low frequency regime.

The key part of the paper is an analysis of the effect of discretization level (i.e. the choice of the number of points at which vibration amplitude measurements are to be taken with the use of accelerometers) on results obtained with the use of the method and their accuracy. The problem of determining an optimum level of discretization for given excitation frequency range is a very important issue as the labor intensity (time-consuming aspect) of the method is one of its main flaws. As far as the technical aspect of the method is concerned, two different geometrical configurations of the measurement setup were tested.
\end{abstract}

Keywords: sound radiation efficiency, sound radiation from plates, Discrete Calculation Method.

\section{Introduction}

The sound radiation efficiency is one of the most useful global physical quantities characterizing radiation generated by a structure vibrating in an elastic fluid. Cases most important from practical point of view include sound radiation induced in air by elements of buildings, machines, etc. A number of methods and techniques were proposed for the purpose of determining frequency dependence of the sound radiation efficiency in both general and specific cases. MAIDANIK (1962) described a statistical method for obtaining acoustic response of a finned plate to excitation with acoustic signal. WALLACE (1970) proposed an asymptotic solution of the problem of radiation resistance generated by a rectangular plate supported freely, mounted in a rigid baffle, and vi- brating in the low frequency range. His approach was based on determination of energy radiated by a single mode to the far field. Both Maidanik (1962) in the above-quoted paper and LEPPINGTON et al. (1982) discussed the foundations of the phenomena related to radiation of sound by structures. Some authors focused their interest on determination of radiation characteristics for axisymmetric vibrating plane structures (RDZANEK et al., 2010; 2012), or discussed the effect of the mutual resistance between individual modes and the sound radiation efficiency, proposing also some improvements in calculation procedures (LI, 2000; 2001). Similar methods of determining the sound radiation efficiency characteristics based on measurements of vibrations of the soundradiating structure were proposed by ARENAS (2009) and Hashimoto (2001). 
The aim of the paper is to examine to what extent the reduction in the amount of measurement points distributed on the plate surface, i.e. discretization applied according to Hashimoto's method, affects the error in evaluation of the sound radiation efficiency. The analysis presented in the paper is important from the practical point of view because the measurements are highly time-consuming. Besides, not always it is necessary to evaluate the sound radiation efficiency in a wide frequency range, and therefore the knowledge of the necessary degree of discretization in different frequency ranges appears to be worth considering. According to Hashimoto, the technique used and analyzed further in the present paper will be called the Discrete Calculation Method (DCM). The physical idea behind DCM consists in dividing the examined soundradiating structure into rectangular elements each of which is further assumed to radiate sound as a rigid hard-baffled piston with the surface area equal to this of the corresponding virtual element.

\section{The sound radiation efficiency}

The sound radiation efficiency, $\sigma$ one of fundamental global physical quantity describes the phenomenon of sound radiation by vibrating structures. It is by definition equal to the total sound power, $W$, generated by a vibrating structure referred to the specific acoustic impedance of the medium in which the sound field is excited, the structure's surface area, and the mean square velocity of its vibrations (FAHY, GARDONIO, 2007):

$$
\sigma=\frac{W}{\rho c\left\langle v^{2}\right\rangle S}
$$

where $W$ is the total sound radiation power, $\rho$ is the density of air, $c$ is the sound speed in air, $\left\langle v^{2}\right\rangle$ is the mean square velocity, and $S$ is the vibrating structure's surface area.

Application of the Discrete Calculation Method proposed by HASHIMOTO (2001) requires that the decision is made regarding division of the vibrating structure (a plate in the present case) into a number of vir-

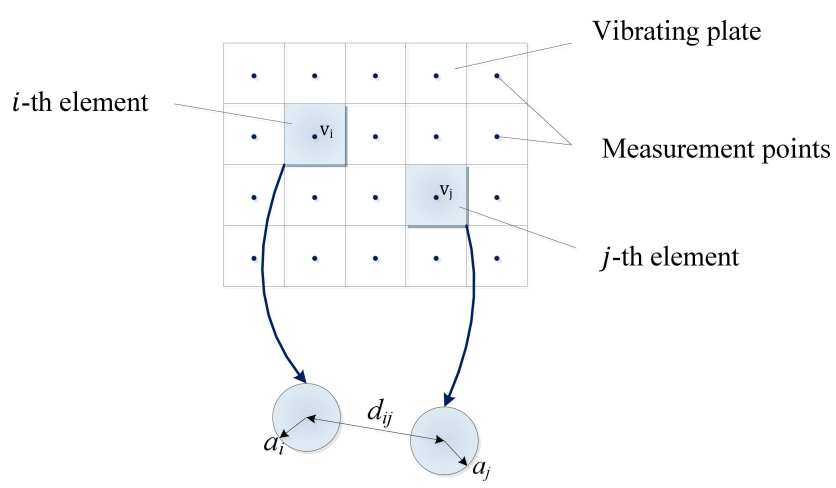

Fig. 1. A diagram illustrating the Discrete Calculation Method. tual elements, usually rectangular. Each of such virtual elementary plates with surface area $s_{i}$ is further assumed to have the same radiation properties as a rigid circular piston with radius

$$
a_{i}=\sqrt{\frac{s_{i}}{\pi}}
$$

The radiation impedance related to each of such elements individually and each mutually interacting pairs of elements can be calculated with the use of formulae obtained by sir Raleigh for impedance of a rigid piston vibrating in an infinite rigid baffle. And so, the self radiation impedance for each of the elements can be calculated as

$$
z_{i i}=\rho c s_{i}\left[1-\frac{J_{1}\left(2 k a_{i}\right)}{k a_{i}}+j \frac{S_{1}\left(2 k a_{i}\right)}{k a_{i}}\right]
$$

while the mutual radiation impedance $z_{i j}$ for each pair of independently vibrating virtual elements can be determined from

$$
\begin{aligned}
z_{i j}= & \frac{\rho c k^{2} s_{i} s_{j}}{2 \pi}\left[\frac{J_{1}\left(k a_{i}\right)}{k a_{i}}\right]\left[\frac{J_{1}\left(k a_{j}\right)}{k a_{j}}\right] \\
& \cdot\left(\frac{\sin k d_{i j}}{k d_{i j}}+j \frac{\cos k d_{i j}}{k d_{i j}}\right),
\end{aligned}
$$

where $k$ is the wave number, $s_{i}$ is the surface area of $i$-th element, $d_{i j}$ is the distance between $i$-th and $j$ th elements, $J_{1}(\cdot)$ is the Bessel function of first order, $S_{1}(\cdot)$ is the Struve function of first order, and $j$ is the imaginary unit. The method of calculating the radiation impedance with the use of the Raleigh integral was expounded in an accessible way by SKUDRZYK (1971).

Vibration measurements are taken at points being centers of elements and the radiation power of individual elements of the plate is calculated as

$$
W_{i}=\operatorname{Re}\left(z_{i i}\right)\left|v_{i}\right|^{2}+\sum_{j} \operatorname{Re}\left(z_{i j} v_{i} v_{j}^{*}\right)
$$

with respective vibration amplitudes $v_{i}$, and further the sound radiation efficiency

$$
\sigma=\frac{\sum_{i} W_{i}}{\rho c\left\langle v^{2}\right\rangle S} .
$$

DCM allows to measure the sound radiation efficiency primarily in the low frequency range. A particularly important merit of the method is the possibility to measure the sound radiation efficiency without necessity to use the standard acoustical apparatus and facilities such as movable microphones operated in anechoic chamber, as the whole information required to calculate the quantity at issue comes from vibration measurements.

НАSнiмото (2001) has extensively discussed correctness of the method and compared it with conventional acoustical techniques. Considerations relating to 
similar rectangular plate dealt with herein is omitted, although relevant analysis was carried out by the present authors in the framework of this study.

\section{The measurement apparatus and setup}

The main structure of the measurement setup was made of 15-mm thick PMMA (acrylic glass) plates. It allowed to mount and measure vibration of rectangular plates with dimensions $500 \times 400 \mathrm{~mm}$ and thickness of about $2 \mathrm{~mm}$. Plates were excited with acoustic signal (white noise) generated by a 10-inch loudspeaker mounted in a diaphragm provided in the mounting structure. Height $l_{z}$ of the transmission chamber could be increased by adding a removable top segment. Contrary to the measurement setup described by НАsнiмото (2001), all internal surfaces were reflecting ones. The plates were mounted on the supporting structure by means of a plastic frame and screws. The measurements were carried out with the use of accelerometers and a signal analyzer in the frequency range $20-1000 \mathrm{~Hz}$, but in view of actual parameters of the loudspeaker used, only results obtained for frequencies higher than $50 \mathrm{~Hz}$ can be considered sufficiently reliable.

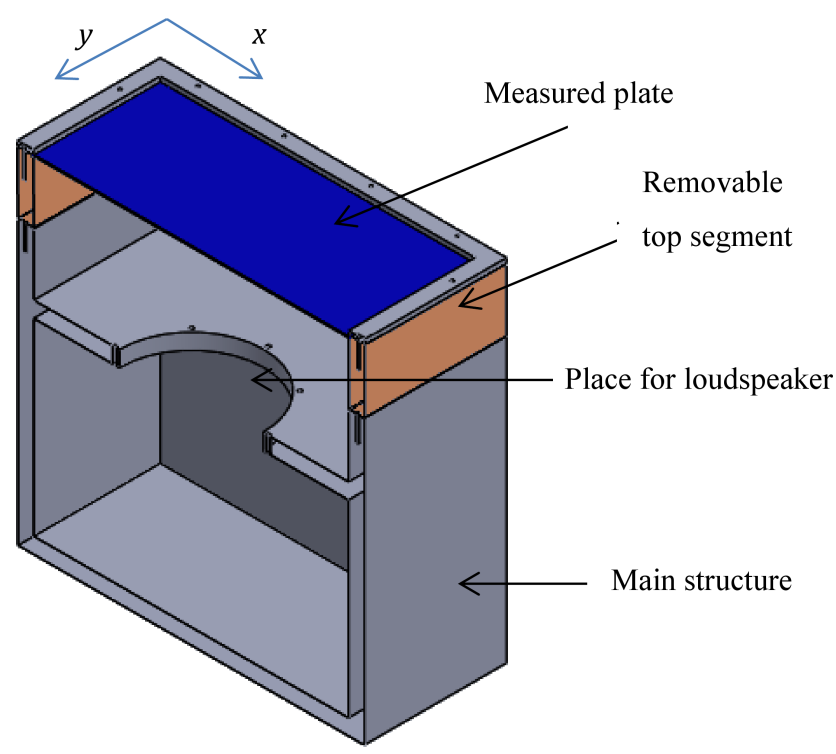

Fig. 2. The measurement setup.

\section{The effect of the transmission chamber height}

The constructed measurement setup allowed to mount examined plates at height $l_{z}=100 \mathrm{~mm}$ or $l_{z}=200 \mathrm{~mm}$ over the loudspeaker mount thus providing a transmitting chamber with variable volume. With the use of such structure, a series of reconnaissance measurements were performed for an aluminum plate $h=2 \mathrm{~mm}$ thick for the measurement setup with transmission chamber with dimensions
$500 \times 400 \times 100 \mathrm{~mm}$ (hereinafter referred to as "cavity A") and $500 \times 400 \times 200 \mathrm{~mm}$ ("cavity B"). Acoustic cavities with such shape, dimensions $l_{x}, l_{y}$, and $l_{z}$, and rigid walls, are characterized with eigenfrequencies (KINSLER, 2000):

$$
f_{l m n}=\frac{c}{2} \sqrt{\left(\frac{l}{l_{x}}\right)^{2}+\left(\frac{m}{l_{y}}\right)^{2}+\left(\frac{n}{l_{z}}\right)^{2}}
$$

values of which for both cavity types are listed in Table 1 .

Table 1. Eigenfrequencies of cavities A and B.

\begin{tabular}{|c|c|c|}
\hline $\begin{array}{c}\text { Vibration mode } \\
(l, m, n)\end{array}$ & \multicolumn{2}{|c|}{ Eigenfrequency value $[\mathrm{Hz}]$} \\
\hline$f_{l m n}$ & $\begin{array}{c}\text { Cavity A } \\
\left(l_{z}=0.1 \mathrm{~m}\right)\end{array}$ & $\begin{array}{c}\text { Cavity B } \\
\left(l_{z}=0.2 \mathrm{~m}\right)\end{array}$ \\
\hline$f_{100}$ & 340 & 340 \\
\hline$f_{010}$ & 425 & 425 \\
\hline$f_{110}$ & 544 & 544.3 \\
\hline$f_{200}$ & 680 & 680 \\
\hline$f_{210}$ & 801 & 801.9 \\
\hline$f_{001}$ & 1700 & 850 \\
\hline$f_{020}$ & 850 & 850 \\
\hline$f_{101}$ & 1733.7 & 915.5 \\
\hline$f_{120}$ & 915 & 915.5 \\
\hline$f_{011}$ & 1752.3 & 950.3 \\
\hline
\end{tabular}

The sound radiation efficiency curves presented in Fig. 3 were obtained for a $2-\mathrm{mm}$ thick aluminum plate and two different heights of the transmission chamber. Very good conformance of the plots can be found up to the frequency of about $145 \mathrm{~Hz}$ at which, on the sound radiation efficiency pattern determined for the setup with cavity A, a maximum occurs absent on the

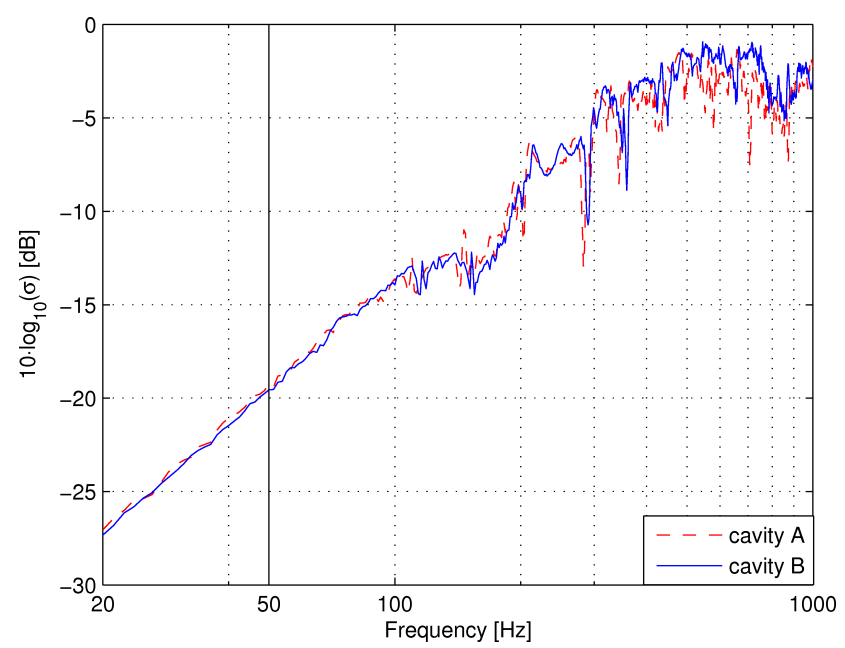

Fig. 3. Values of the sound radiation efficiency determined for 2-mm thick aluminum plate using the measurement setup with two different transmission chamber heights. 
graph plotted for type B transmitting chamber. This frequency is equal to analytically calculated value of the eigenfrequency corresponding to mode $(1,2)$ of a freely-supported plate with the same parameters. Similar maximum and minimum peaks appeared also in several other series of measurements taken in the course of the study but it turned out to be impossible to find any straightforward relationship between these features and either the vibration velocity distribution on the plate surface or parameters of the measurement procedure and setup (i.e. number of measurement points, plate material, transmission chamber height, etc.).

More significant discrepancies between the curves can be observed for higher frequencies; however, they seem to follow not only from different height of the transmission chamber but also from the adopted number of measurement points lower than this recommended for the examined vibration frequency range and, additionally, from problems with providing sufficiently repeatable plate mounting system. In further stages of the study, measurements were performed on the setup with the transmission chamber height fixed at $l_{z}=0.1 \mathrm{~m}$.

\section{The effect of the number of measurement points}

According to recommendations provided by Hashimoto (2001), the maximum size of a virtual plate element should not exceed a half of wavelength corresponding to the coincidence (critical) frequency. Determination of the sound radiation efficiency for plates characterized with high coincidence frequency means that large number of vibration velocity measurements must be taken which makes the method very labor intensive. Taking into account the fact that the low frequency regime is of primary interest in the majority of cases relating to practical applications, the present study was focused on examining what would be the effect of reduced number of measurement points on quality of the obtained results. Figure 4 presents plots of the sound radiation efficiency obtained for the aluminum and the steel plate, both 2-mm thick, as a result of calculations based on vibration measurements taken at grids of $320,80,35$, and 12 points. According to the Hashimoto's criterion, for the examined aluminum plate characterized with the coincidence frequency of $5865 \mathrm{~Hz}$, the largest dimension of the virtual element should not exceed $0.029 \mathrm{~m}$, compared to $0.02 \mathrm{~m}$ for the steel plate for which the coincidence frequency is $6003 \mathrm{~Hz}$. The condition was met for both plates in the case of measurements at 320 points (cf. Table 2). Curves in Fig. 4 obtained for 320 points are plotted as a reference for each of the less dense measurement grids.
Table 2. Numbers of measurement points adopted in the study and corresponding maximum dimensions of virtual elements in $x$ and $y$ direction.

\begin{tabular}{|c|c|c|}
\hline \multirow{2}{*}{$\begin{array}{c}\text { Number } \\
\text { of measurement points }\end{array}$} & \multicolumn{2}{|c|}{ Maximum element dimensions } \\
\hline & $\begin{array}{c}x \text {-direction } \\
{[\mathrm{mm}]}\end{array}$ & $\begin{array}{c}y \text {-direction } \\
{[\mathrm{mm}]}\end{array}$ \\
\hline 320 & 25 & 25 \\
\hline 80 & 50 & 50 \\
\hline 35 & 71 & 80 \\
\hline 12 & 125 & 133 \\
\hline
\end{tabular}

It can be seen that the sound radiation efficiency curves calculated from measurement data taken at points of less dense grids are, at least for the aluminum plate, consistent with those obtained from a series of 320 individual measurements in the low frequency regime (Fig. 4a). With increasing virtual element size, the frequency up to which different curves represent values close to each other decreases. For the steel plate (Fig. 4b), the trend is roughly the same. It should be also noted that results obtained for frequencies lower than $50 \mathrm{~Hz}$ can be biased with a significant error in view of a limited transmission band characterizing the used loudspeaker, although it must be concluded that the presented plots demonstrate deviation from the expected slope ( $6 \mathrm{~dB}$ per octave) only for the steel plate and only for 320 measurements taken on it.

Figure 5 presents the relative error of evaluation of the sound radiation efficiency calculated in the wide frequency range and referred to the most dense discretization of the plate surface (320 measurement points). For the number of discretization points equaling 12,35 , and 80 , these errors remained in a ratio of 281:157:87 for the examined aluminum plate and 270:197:118 for the steel plate, respectively. As it was expected, the error increases as the discrimination decreases.

To illustrate the difference resulting from the increase of distance between measurement points, Figs. 6 and 7 present plots of the sound radiation efficiency versus frequency with its values divided into components coming from the self radiation power of individual plate elements (considered as circular pistons with surface areas equal to these of corresponding virtual rectangles) on one hand and the mutual radiation power corresponding to all pairs of elements on the other. Additionally the figures, of which Fig. 6 and Fig. 7 present results obtained for aluminum and steel plate, respectively, contain plots of the sound radiation efficiency calculated theoretically for a circular piston with the surface area equal to this of the whole plate.

The plot representing the sound radiation efficiency for an aluminum plate determined as a result of measurements taken at 80 points in the frequency range from $20 \mathrm{~Hz}$ to about $340 \mathrm{~Hz}$ is very close to the curve 
a)
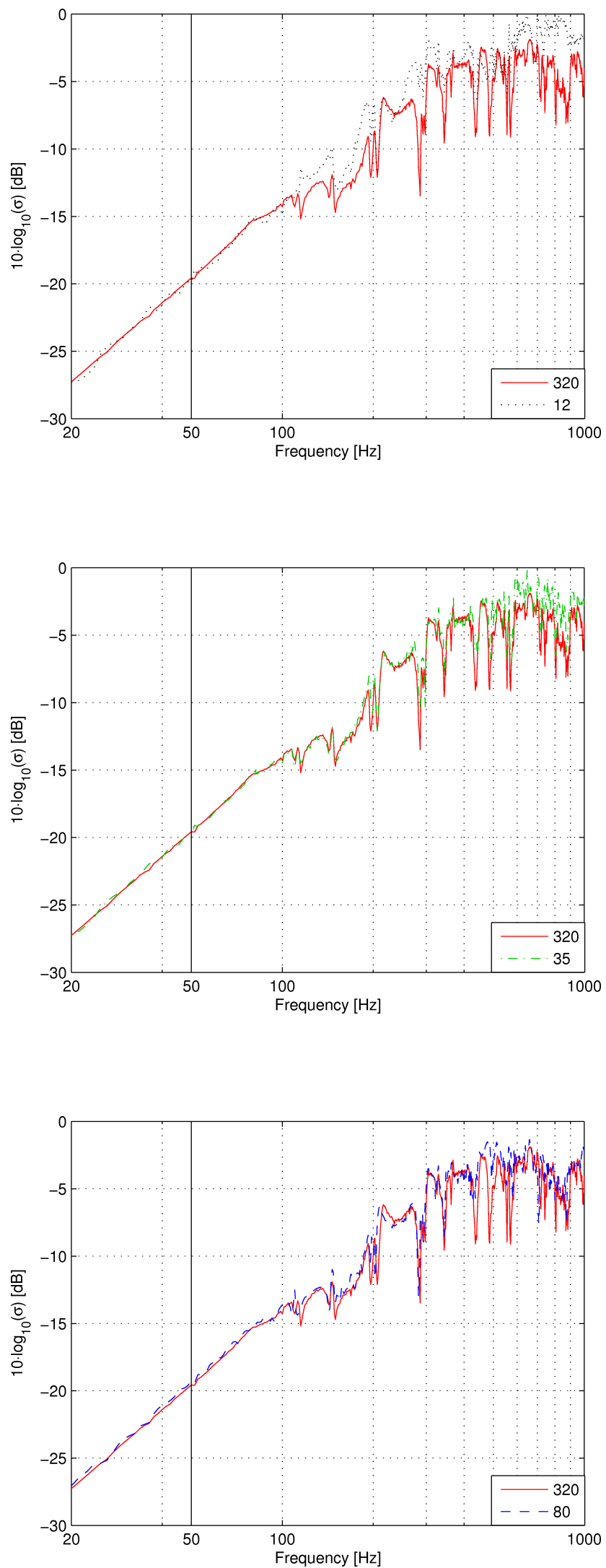

b)
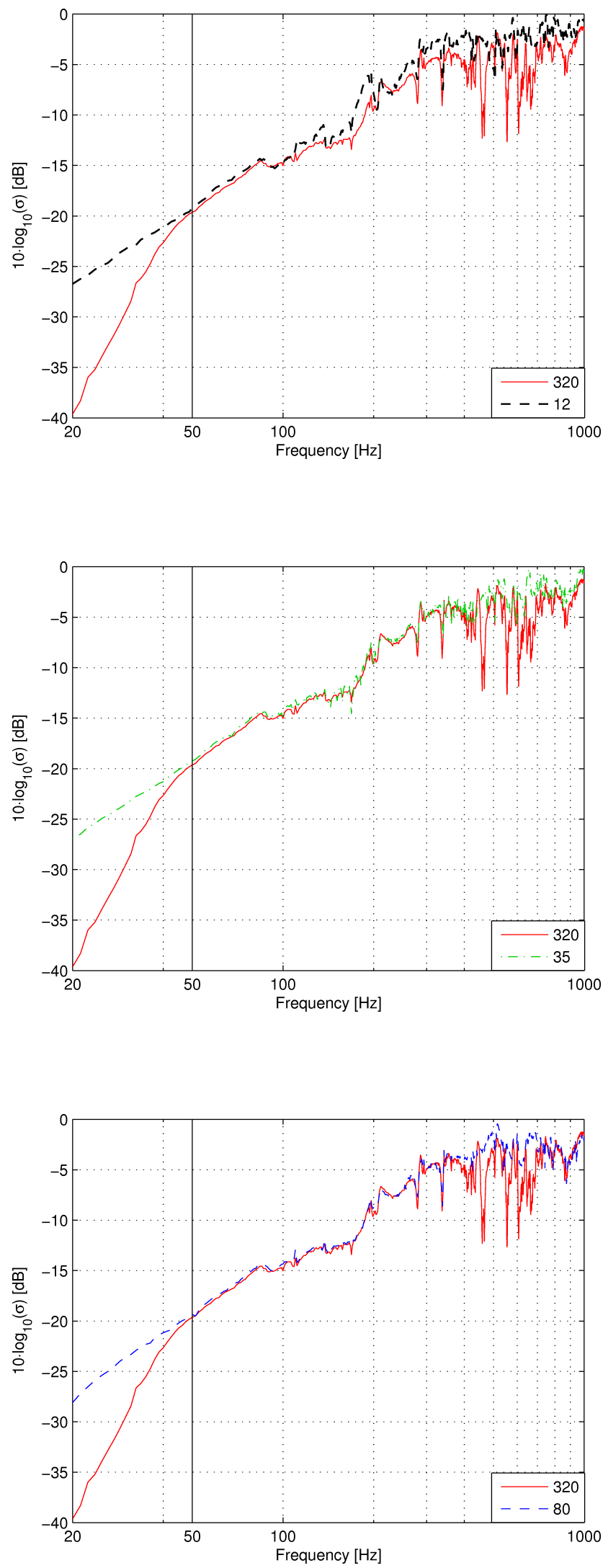

Fig. 4. Values of the sound radiation efficiency determined for (a) aluminum and (b) steel plate with vibration measurements taken at different numbers of points. The curve obtained for 320 points is plotted as a reference for each of the less dense measurement grids. 
a)

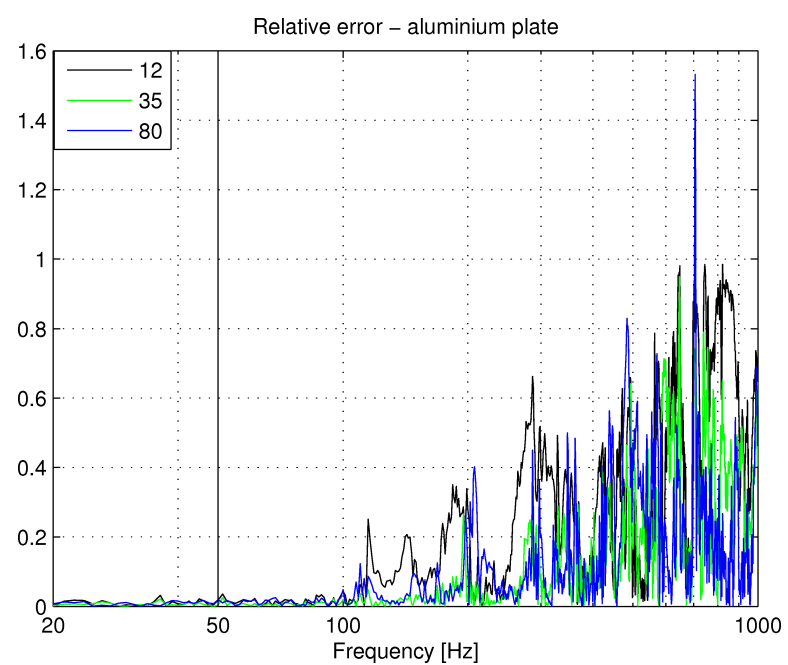

b)

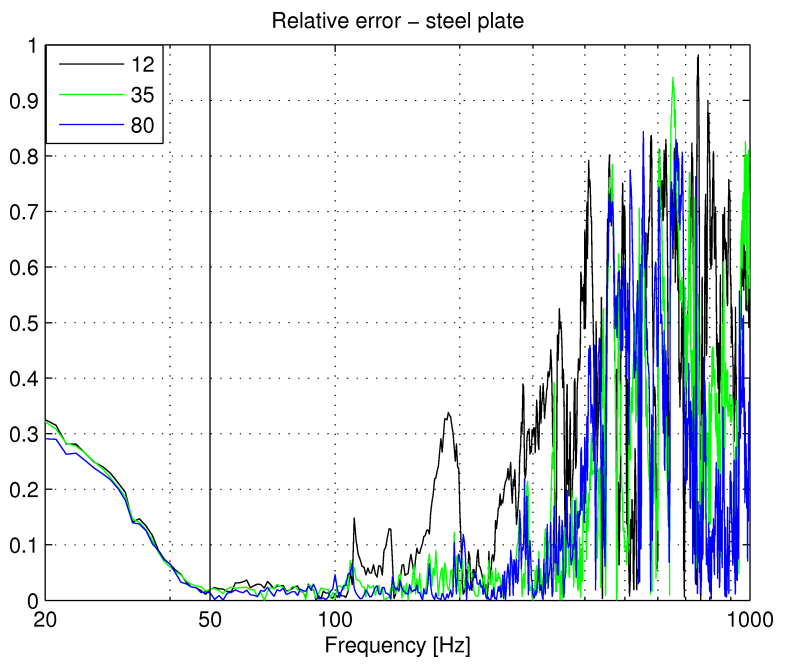

Fig. 5. Relative error of determining the sound radiation efficiency value depending on plate discretization
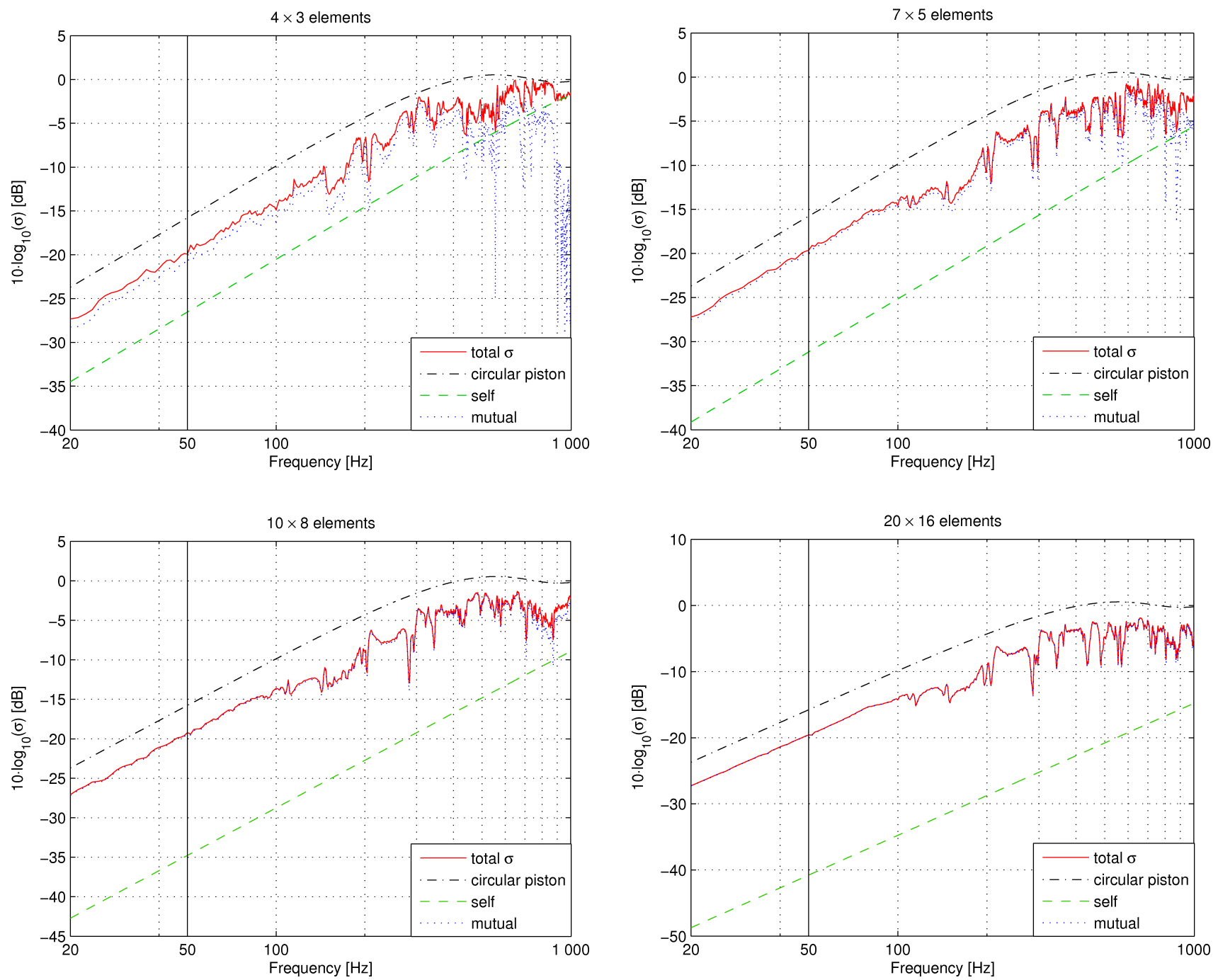

Fig. 6. Plots representing components of the sound radiation efficiency for a 2-mm thick aluminum rectangular plate coming from the self power and the mutual power compared to the impedance of circular piston with the same surface area. 

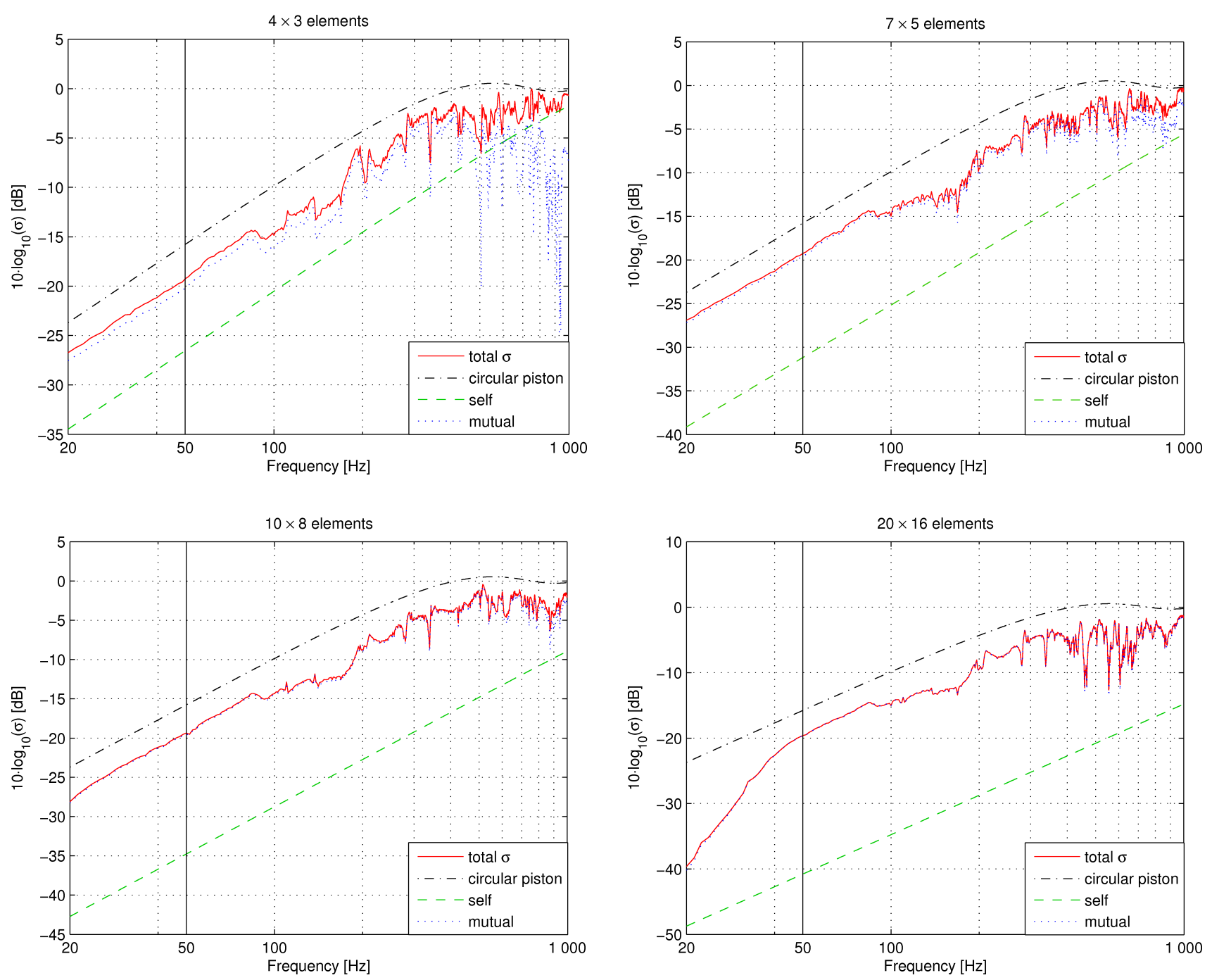

Fig. 7. Same as Fig. 6 but for a 2-mm thick steel plate.

calculated with the use of vibration velocity measurements made at a grid of 320 points. Minor differences may follow from the fact that the series of measurements involving different number of points were not taken immediately one after another - the plate was dismounted from the setup structure and later mounted again. However, for measurements taken at 80 points, no rapid drops in the sound radiation efficiency are observed at frequencies corresponding to eigenfrequencies of the cavity, such as e.g. $436.25 \mathrm{~Hz}$, $555 \mathrm{~Hz}$, or $806.25 \mathrm{~Hz}$ (cf. Table 1).

For the steel plate, the obtained sound radiation efficiency curves coincide in the frequency range from $50 \mathrm{~Hz}$ to about $340 \mathrm{~Hz}$. The measurements carried out at points of grids with different density have been carried out without dismounting the plate and thus the results were free from interference related to possible change of boundary conditions. In Figs. 6 and 7, a characteristic feature can be observed: with increasing virtual element size, the frequency for which the curve representing the sound radiation efficiency component coming from the acoustic self power crosses the curve depicting the radiation coefficient component related to the mutual component of the radiated sound power decreases. For aluminum plate and 320 measurement points, such crossing point falls outside the adopted frequency range; for 80 points it occurs at $870 \mathrm{~Hz}$, for 35 points it appears at $573 \mathrm{~Hz}$, and for 12 measurement points - at $206 \mathrm{~Hz}$. Mutual component of the elements' sound radiation power dominates for lower frequencies, and with increasing frequency, the share of self radiation generated by the elements increases.

The comparative analysis presented above allows to state that when the subject of interest if the sound radiation efficiency in the low frequency range, it can be determined by taking vibration velocity amplitude measurements at points located in centers of virtual elements maximum dimension of which is less than a half of wavelength corresponding to the coincidence frequency. The use of the method is also justified when the objective of the measurement is a preliminary estimation of the sound radiation efficiency value. On the 
grounds of tests carried out in the framework of the present study it can be claimed that reliable results can be obtained using DCM for frequencies lower than the crossover point of curves representing self radiation and mutual radiation components of the power generated by the plate.

The performed measurements prove that satisfactory results are obtained for frequencies lower than a characteristic frequency at which the curves representing the plate's self radiation power on one hand and mutual radiation power on the other cross each other (cf. Figs. 6 and 7).

Among possible sources of errors specific for the used measurement setup and procedure, one should mention, above all, insufficient repeatability of actual boundary conditions related to mounting and dismounting the examined plates. Further, for reasons concerning nature of the mounting structure, it was impossible to avoid certain unevenness of the pressure exerted on plate edges around its perimeter by screws used as fasteners, despite due care taken when tightening them. Another factors potentially affecting accuracy of the obtained results are resonance phenomena occurring in the mounting structure and inevitable change of plate properties resulting from attaching accelerometers to its surface.

\section{Conclusions}

A series of vibration amplitude measurements in the frequency range of $50-1000 \mathrm{~Hz}$ have been carried out on aluminum and steel plates using a dedicated mounting setup. Measurement points on plates with dimensions $500 \times 400 \times 2 \mathrm{~mm}$ form grids of various density. Using the Discrete Calculation Method proposed by HАsнiмото (2001), values of the sound radiation efficiency were calculated from the obtained discrete vibration velocity amplitude data.

The conclusions are as follows:

- Tests carried out with two different geometries of the measurement setup allowed to state that the height of the transmission chamber had no significant effect on accuracy of the adopted method of determining the sound radiation efficiency of plates.

- Possible practical applications of the DCM technique can be implemented where the sound radiation efficiency values for low frequencies are usually of the greatest interest. For the purpose of examining the low frequency range, the effect of increasing the maximum linear dimension of the virtual plate element above the recommended value equaling a half of the wavelength corresponding to the coincidence frequency has been examined.

- In a series of tests, dimension of the virtual element was increased from $25 \mathrm{~mm}$ (the length meeting the Hashimoto's criterion) successively to $0.05 \mathrm{~m}, 0.08 \mathrm{~m}$, and $0.13 \mathrm{~m}$, which resulted in progressing reduction of time required to take necessary number of vibration measurements. Analysis of the obtained results allows to draw a conclusion that also in these cases of reduced measurement grid density, correct values of the sound radiation efficiency can be obtained for the frequency range below the crossing point of curves representing the self radiation power and the mutual radiation power.

- If measurements are taken at a relatively lower number of points, information about resonance features is being lost; however, where the objective of the measurement is a preliminary estimation of the sound radiation efficiency value, such reduction of the discretization degree may prove to be grounded well enough.

\section{References}

1. Arenas J.P. (2009), Matrix method for estimating the sound power radiated from a vibrating plate for noise control engineering applications, Latin American Applied Research, 39, 345-352.

2. Fahy F., Gardonio P. (2007), Sound and Structural Vibration - Radiation, Transmission and Response, Amsterdam etc., Academic Press 2007, 135-240.

3. Hashimoto N. (2001), Measurement of sound radiation efficiency by the discrete calculation method, Applied Acoustics, 62, 429-446.

4. Kinsler L.E., Frey A.R., Coppens A.B., SanDERS J.V. (2000), Fundamentals of Acoustics, Fourth Edition, New York, John Wiley \& Sons, Inc., 246-248.

5. Leppington F.G., Broadbent E.G., Heron K.H. (1982), The Acoustic Radiation Efficiency of Rectangular Panels, Proc. R. Soc. Lond. A, 382, 245-271.

6. Li W.L. (2001), An analytical solution for the selfand mutual radiation resistances of a rectangular plate, Journal of Sound and Vibration, 245, 1, 1-16.

7. Maidanik G. (1962), Response of Ribbed Panels to Reverberant Acoustic Fields, J. Acoust. Soc. Amer., 34, 6, 809-826.

8. Rdzanek W.P., Rdzanek W.J., Szemela K. (2010), Asymptotic approximation of the modal acoustic impedance of a circular membrane, Journal of Computational Acoustics, 18, 4, 335-362.

9. Rdzanek W.P., Rdzanek W.J., Pieczonka D. (2012), The Acoustic Impedance of a Vibrating Annular Piston Located on a Flat Rigid Baffle Around a SemiInfinite Circular Rigid Cylinder, Archives of Acoustics, 37, 4, 411-422.

10. SkudrzyK E. (1971), The Foundations of Acoustics Basic Mathematics and Basic Acoustics, New YorkWien, Springer-Velag, 663-676.

11. Wallace C.E. (1970), Radiation Resistance of Rectangular Panel, J. Acoust. Soc. Amer., 51, 946-952. 